PROFESSOR KRISTIAN GUNDERSEN (Orcid ID : 0000-0001-9040-3126)

Article type : Regular Paper

\title{
Specific labelling of myonuclei by an antibody against Pericentriolar material 1 (PCM1) on skeletal muscle tissue sections
}

Running title: Myonuclei can be specifically labelled by PCM1

Authors:

I. M. Winje, ${ }^{1}$ M. Bengtsen, ${ }^{1}$ E. Eftest $\varnothing 1,{ }^{1}$ I. Juvkam, ${ }^{1}$ J. C. Bruusgaard ${ }^{1,2}$ and K. Gundersen, ${ }^{1, *}$

${ }^{1}$ Department of Biosciences, University of Oslo, NO-0316 Blindern, Oslo, Norway

${ }^{2}$ Department of Health Sciences, Kristiania University College, P.O. Box 1190 Sentrum, NO-

0107 Oslo, Norway

*Corresponding author:

kristian.gundersen@ibv.uio.no

\section{Abstract}

Aim: Skeletal muscle is a heterogeneous tissue containing several different cell types and only about $40-50 \%$ of the cell nuclei within the tissue belong to myofibres. Existing technology, attempting to distinguish myonuclei from other nuclei at the light microscopy

This article has been accepted for publication and undergone full peer review but has not been through the copyediting, typesetting, pagination and proofreading process, which may lead to differences between this version and the Version of Record. Please cite this article as doi: 10.1111/apha.13034

This article is protected by copyright. All rights reserved. 
level, has led to controversies in our understanding of the basic cell biology of muscle plasticity. The present study aims at demonstrating that an antibody against the protein Pericentriolar material 1 (PCM1) can be used to reliably identify myonuclei on histological cross sections from humans, mice and rats.

Methods: Cryo-sections were labelled with a polyclonal antibody against PCM1. The specificity of the labelling for myonuclei was verified by using 3D reconstructions of confocal z-stacks triple labelled for DNA, dystrophin and PCM1, and by co-localization with nuclear mCherry driven by the muscle specific Actin Alpha 1 promoter after viral transduction.

Results: The PCM1 antibody specifically labelled all myonuclei, and myonuclei only, in cryo-sections of muscles from rats, mice and men. Nuclei in other cell types including satellite cells were not labelled. Both normal muscles and hypertrophic muscles after synergist ablation were investigated.

Conclusion: PCM1 can be used as a specific histological marker for myonuclei in skeletal muscle tissue without relying on counter staining of other structures or cumbersome and subjective analysis of nuclear positioning.

Keywords: myonuclei; hypertrophy; PCM1; skeletal muscle; satellite cells

\section{Abbreviation list}

AAV-6, Adeno Associated Vector zerotype-6; ACTA1, Actin, Alpha 1, Skeletal Muscle; DPBS, Dulbecco's phosphate-buffered saline; EDL, extensor digitorum longus; EM, electron microscopy; NLS, nuclear localization signal; PCM1, pericentriolar material 1; TA, tibialis anterior

This article is protected by copyright. All rights reserved. 


\section{Introduction}

Most tissues are heterogeneous and this represents an inherent problem when analysing homogenates or even histological sections at the light microscopy level. The latter has been solved in many systems by using cell-specific or organelle-specific antibodies.

Skeletal muscle tissue is also heterogeneous and contains long multinucleated cells called myofibres, fibroblasts in the connective tissues, various cells in the blood vessels and the myogenic stem cells called satellite cells. The satellite cells are the source of new myonuclei for growing and regenerating muscle fibres, and were originally identified by electron microscopy (EM) ${ }^{1}$. They are particularly difficult to distinguish from the myonuclei since they reside between the basal lamina and cell membrane of the muscle fibres.

Only the nuclei inside the muscle fibre sarcolemma contribute to their protein expression and are defined as true myonuclei. Depending on the muscle being analysed, electron microscopy has revealed that only approximately $40-50 \%$ of the nuclei found in muscle tissue are myonuclei ${ }^{2}$, but so far there has been no generally accepted immunohistochemical marker to discern these nuclei at the light microscopy level.

While $\mathrm{EM}^{2}$, and in vivo imaging ${ }^{3-9}$, can be used to reliably identify myonuclei, analysis of tissue sections using regular light microscopy has led to conflicting results in the understanding of how myonuclei are related to cell size regulation as we have discussed previously 10,11

The standard model for size regulation has implied that when muscle fibres grow or hypertrophies satellite cells fuse to the enlarged fibres to support the larger cytoplasm. Conversely, during atrophy some of the myonuclei inside the intact fibres are allegedly lost by a nuclear apoptotic process as the cell is returning to a state of a smaller calibre with fewer nuclei (reviewed in ${ }^{12}$ ).

This model is to a large extent based on histology with classical chemical staining methods and has recently been challenged by direct observation of myonuclei by in vivo imaging (reviewed in ${ }^{10,13}$ ). The new results indicate that recruitment of myonuclei precedes cell enlargement ${ }^{7}$, and is obligatory at least for major de novo hypertrophy ${ }^{9,14,15}$, and in direct conflict with the old model, myonuclei are not lost during atrophy ${ }^{6,11}$. The "extra" nuclei that have been acquired by previous hypertrophy seem to be permanent and are aiding in a more

This article is protected by copyright. All rights reserved. 
rapid subsequent regrowth. This was the foundation of the recent proposal of a cellular memory in muscle ${ }^{8}$.

Using dystrophin staining to label the fibre cortex and determining which nuclei are within the dystrophin ring of fibre cross sections can result in data consistent with those obtained by in vivo imaging. But even this approach has led to conflicting findings ${ }^{16-18}$. In order to identify myonuclei specifically, a strict and cumbersome evaluation procedure for each nucleus should be used. This has not been adhered to in most of the literature, and even when it is, the method is very sensitive for small and subjective differences. Thus, in our laboratory when the same unbiased operator evaluated the same sections, but were instructed to count only the nuclei with their geometric centre inside the dystrophin ring, the number of nuclei was significantly reduced compared to the number obtained when also nuclei with their centre on the ring was included ${ }^{11}$.

In our previous histological studies, we have consistently defined myonuclei as nuclei with their geometrical centre inside the inner rim of the dystrophin ring ${ }^{6,7,10,11}$. While this has given results consistent with in vivo imaging data, we have been worried that the criterion is too strict and that some myonuclei were not identified as such (false negatives).

In dividing cells the protein Pericentriolar material 1 (PCM1) is associated with the centrosome where it is important for microtubule organization and centrosome proteostasis ${ }^{19-}$ ${ }^{21}$. In differentiated post-mitotic myocytes, the protein re-localizes to the nuclear envelope where it forms an insoluble matrix ${ }^{22-24}$. As myonuclei are in a post-mitotic state, PCM1 is found on the nuclear envelope in adult skeletal muscle ${ }^{23,24}$. An antibody against PCM1 has been used for identifying cardiomyocyte nuclei ${ }^{25}$, and we hypothesized that PCM1 could be used as a novel marker to identify skeletal muscle myonuclei at the light microscopy level.

We report here that PCM1 specifically labelled myonuclei, and not the nuclei belonging to satellite cells or other cell types in human and rodent muscle tissue.

This article is protected by copyright. All rights reserved. 


\section{Results}

\section{PCM1 selectively labeled myonuclei}

To investigate if PCM1 could be used as a marker for myonuclei, we stained cross sections of skeletal muscles from humans, mice and rats with DAPI, dystrophin, and PCM1. Detailed inspection of the sections in several confocal planes showed that all nuclei inside the dystrophin ring were positive for PCM1 in all three species. The confocal stack analysis was performed on 689 nuclei from 3 mice (Figure 1) 323 nuclei from 4 humans (Figure 2a-c), and 286 nuclei from 3 rats (Figure 2d).

In some cases, separating individual nuclei was difficult, especially when they were partly overlapping, but in different z-planes. An example is shown in Figure 1b. Inspection of their position using confocal 3D-rendering revealed that these were indeed separate nuclei, one being labelled for PCM1 (Figure 1c). In some cases, PCM1 positive nuclei appeared to be outside the dystrophin ring (Figure $1 \mathrm{~b}$ and $2 \mathrm{~b}$ ), but closer inspection of their position using confocal z-stacks confirmed that they were indeed situated inside the dystrophin ring (Figure 1c and 2c). Using these methods for screening a total of 1298 PCM positive nuclei from 13 muscles in the three species, we did not find any PCM1 positive nuclei that appeared to be outside the dystrophin ring, and all the DAPI positive nuclei inside the ring co-stained for PCM1.

We then randomly selected 3 random images from the data material in Figure 1 and all DAPI-positive nuclei-structures were evaluated for PCM1 labelling. In EDL and soleus 1034 and 1129 DAPI positive structures were scrutinized in this way, excluding veins and large non-myofibrillar bodies. We found that $50 \%$ and $46 \%$ of the DAPI positive structures were PCM1 positive in EDL and soleus respectively. Such fractions of myonuclear nuclei of the total number of nuclei are similar to those inferred from EM images from fast and slow muscles $^{2}$.

Finally, we used the same images to compare PCM1 labelling and a purely positional method. As described in the Introduction we have previously defined myonuclei as nuclei with their geometrical centre inside the inner rim of the dystrophin ring as judged by nonconfocal imaging 6,7,10,11. We here used the same method using one confocal plane. When analysed in this way we deemed 248 and 314 nuclei to fill the positional criteria for a

This article is protected by copyright. All rights reserved. 
myonucleus, while 287 and 348 nuclei were PCM1 positive in EDL and soleus respectively. Thus, the number of PCM1 positive nuclei was $16 \%$ and $11 \%$ higher (Figure 3a). When the discordant nuclei were analysed in several focal planes, it was confirmed that all these PCM1 positive nuclei were indeed inside the dystrophin ring (Figure $3 \mathrm{~b}$ and $3 \mathrm{c}$ ). This suggests that the purely positional method is too conservative when only one image plane is used, e.g. leads to false negatives.

\section{PCM1 co-localized with myonuclei-specific mCherry expression}

To further validate that PCM1 labels all myonuclei, we investigated the localization of PCM1 by the aid of viral transduction of a mCherry construct fused to a nuclear localization signal peptide under the control of the muscle specific Actin alpha 1 (ACTA1) promoter (Figure 4a). Analysis of cross sections stained for dystrophic and visualised for mCherry, showed that we transduced approximately $20 \%$ of the muscle fibres. Of 165 nuclei ( $n=3$ mice) labelled with mCherry, all were found inside of the dystrophin ring and hence were myonuclei (Figure 4b). This was followed by inspection of the co-localization of PCM1 and mCherry revealing that all mCherry-verified myonuclei were found to be positive for PCM1 staining (Figure 4c).

In addition, the PCM1 antibody labelled a band with the size corresponding to PCM1 (Figure 4D) ${ }^{26}$ Additional analyses of the soluble and insoluble fraction of the muscle lysate showed that PCM1 localized to the insoluble fraction, consistent with the interpretation that PCM1 is localized to the nuclear membrane ${ }^{23}$.

\section{PCM1 did not label satellite cells}

Satellite cells are activated during repair and hypertrophy, and will then increase in number as a source for new myonuclei. We were concerned that PCM1 might label satellite cells and give rise to false positives. Additionally, the intimate association with the sarcolemma makes it difficult to discern these nuclei by using positional methods. On sections triple-labelled for PCM1, PAX7 and DNA we screened 24 PAX7 positive nuclei, and none of them stained for PCM1 (Figure 5). For this experiment overloaded muscles were used in order to increase the number of satellite cells (PAX7-positive), as during basal conditions the number of PAX7 positive satellite cells detected on muscle cross sections is very low.

This article is protected by copyright. All rights reserved. 


\section{PCM1 labelled all the myonuclei of overloaded muscles}

We next investigated whether the PCM1 antibody would also label myonuclei inserted during hypertrophy by overloading soleus by synergist ablation ${ }^{8}$.

Muscle cross sections were stained with the PCM1 antibody after 14 days of overload, revealing a $16 \%$ increase in PCM1-positive nuclei and a $14 \%$ increase in fibre cross-sectional area compared to contralateral control muscles (Figure 6). Like in naive muscles, essentially all the nuclei inside the dystrophin ring were PCM1 positive. This indicates that the PCM1 antibody also labelled the newly recruited myonuclei under these experimental conditions.

\section{Discussion}

In the present study, we show that a PCM1 antibody specifically labels all myonuclei and myonuclei only in cryo-sections of rats, mice and men. Importantly, by Pax7 co-staining it was determined that PCM1 did not label satellite cells which are particularly difficult to discern due to its close proximity to the muscle fibres. Thus PCM1 can be used as a histological marker for this subset of nuclei in skeletal muscle tissue without relying on counterstaining of other structures or cumbersome and subjective analysis of nuclear positioning sometimes at the limit of the microscopical resolution.

There has been no established gold standard for identifying myonuclei in histological sections, but the best practice has been based on labelling all the nuclei in the tissue with a DNA stain, and then identifying the myonuclei by their position relative to the dystrophin labelling of the fibre cortex cytoskeleton (see for example ${ }^{6-11,16,17,18,27-29}$ ).

Besides the subjectivity in assessing the position of the myonuclei, our confocal analysis demonstrate that small deviations in the angle of the nuclei relative to the section plane may result in individual nuclei appearing to be positioned in, on or outside the fibre boundary, and since dystrophin localizes in distinct sub-sarcolemmal domains in mammalian skeletal muscle, local folds and tissue conditions may result in a broader appearance of the dystrophin ring when being analysed on sections ${ }^{30}$.

The number of PCM1 labelled nuclei was 11-16\% higher than the number of myonuclei observed with their geometric centre inside the inner rim of the dystrophin ring by nonconfocal imaging. By a detailed analysis of several confocal planes through the sections it

This article is protected by copyright. All rights reserved. 
was confirmed that these nuclei were in fact also inside the dystrophin ring, and that the inclusion criterion using geometric centres was too strict so that not all myonuclei were included, e.g. false negatives. While false negatives might distort absolute numbers, they probably represent a random subpopulation of nuclei, and would not distort conclusions about myonuclear behaviour.

In most of the existing literature no explicit precise criteria for determining the nuclear position nor a closer 3D evaluation has been used, and this might have led to inclusion of nuclei that were not true myonuclei, i.e. false positives. This is a more serious problem than false negatives, since it would include nuclei in other cell types with a different biology. For example, it might have led to a false notion that myonuclei undergo apoptosis during disuse atrophy (for references see ${ }^{10}$ ), while in fact more precise methods indicate that the increase in apoptotic behaviour is limited to other cell types in the muscle tissue while myonuclei seems to be protected ${ }^{6,10,11}$. PCM1 labelling will provide a new simple tool for avoiding false identification of myonuclei in a complex tissue.

In conclusion, PCM1 staining provides a new simple and robust method for selectively and reliably labelling myonuclei in rodents and humans, eliminating the need for a detailed and often subjective assessment of the position of each nucleus near the limit of the resolution for conventional light microscopy.

\section{Methods}

\section{Ethical approval}

\section{Animal studies}

Female NMRI mice weighing 28-31 grams or male Sprague Dawley rats weighing 210-260 grams (613-660g for overload experiment) were used. Animals were kept at the animal facility at the Department of Biosciences, University of Oslo. Animals were housed with a 12 $\mathrm{h}$ light/dark cycle with ad libitum access to food and water. For non-terminal experiments, animals were sedated with inhalation gas anaesthesia with $2 \%$ isoflurane (506949, Forene, Abbot) in air. For terminal experiments, animals were sacrificed by cervical dislocation while under deep anaesthesia. Research was conducted in accordance with the Norwegian Animal Welfare Act of 20th December 1974, and all experiments were approved by The Norwegian Animal Research Committee before initiation. The Norwegian Animal Research Authority

This article is protected by copyright. All rights reserved. 
provided governance to ensure that facilities and experiments were in accordance with the Act, National Regulations of January 15th, 1996, and the European Convention for the Protection of Vertebrate Animals Used for Experimental and Other Scientific Purposes of March 18th, 1986.

Human studies

For human studies The Regional Committees for Medical and Health Research Ethics in Southeast Norway approved the study (2014/834), which was performed in accordance with the Declaration of Helsinki. All subjects signed a written informed consent prior to study participation.

\section{Transduction using recombinant Adeno Associated Virus zerotype-6}

To visualize myonuclei by an independent method a custom AAV6 vector comprised of the human Actin, Alpha-1 (ACTA1) gene promoter and nuclear mCherry was constructed. The promoter region (3111 bp to 740 bp upstream of the transcription start site) for the ACTA1 gene was amplified by PCR and sub cloned into the pCherry vector carrying a nuclear localization signal (NLS) upstream of the mCherry cassette. The expression cassette was then cloned into the pAAV6-Basic vector. The final construct was validated by Sanger sequencing (results not shown). Virus carrying the ACTA1-Vector was produced by Vector Biolabs. For transduction, 6-week-old female NMRI mice (Janvier Labs, France) was injected with $5 \times 10^{\wedge} 9$ genome copies of Adeno-Associated Virus 6 in $20 \mu 1$ sterile Dulbecco's phosphatebuffered saline (DPBS). 14 days after transduction Tibialis anterior (TA) muscles were isolated as described below.

\section{Functional overload by synergist ablation}

Overload of rat plantaris and soleus muscles was induced by excising the distal $1 / 3$ of the gastrocnemius, leaving all neural- and blood- supply to plantaris and soleus intact. Ablations were performed unilaterally, whereas the left foot not undergoing overload served as a contralateral control. 14 days after synergist ablation the plantaris and soleus muscles were isolated as described below.

This article is protected by copyright. All rights reserved. 


\section{Muscle isolation and sectioning}

Muscles from 8-week-old untreated (Figure 1 and 3) or transducted (Figure 4) female NMRI mice, and untreated 8-week-old (Figure 2) or 21-week-old overloaded male Sprague Dawley rats (Figure 5-6) were isolated, embedded in OCT Tissue-Tek (Sakura Finetek Europe B.V.), frozen slightly stretched in melting iso-pentane (Sigma-Aldrich) and stored at $-80{ }^{\circ} \mathrm{C}$ followed by cryo-sectioning at 20 or 10 (overload exp.) $\mu \mathrm{m}$. Human cryo-sections $(20 \mu \mathrm{m})$ was kind gift from Dr. Kristoffer Toldnes Cumming, Norwegian School of Sport Sciences, Oslo, Norway.

\section{Histochemistry}

\section{PCM1}

Sections retrieved from $-80{ }^{\circ} \mathrm{C}$ were allowed to equilibrate for 30 minutes at room temperature. Before staining, slides were pre-incubated with 2\% BSA in PBS pH 7.4 for 30 min. Sections were stained with a Rabbit polyclonal antibody against PCM1 (1:1000 HPA023370, Sigma) in staining solution (5\% BSA in PBS, 0.2\% Igepal CA-630) overnight at $4{ }^{\circ} \mathrm{C}$. Next day the sections were washed three times for $5 \mathrm{~min}$ in PBS, and stained with an anti-Rabbit secondary antibody (1:1000, ab150077, Abcam) in $2 \%$ BSA in PBS for 1 hour. Sections were washed three times for 10 min with PBS. For visualizing dystrophin, the sections were stained with supernatant containing a mouse monoclonal antibody diluted in staining buffer (1:20, MANDYS8(8H11), Developmental Studies Hybridoma Bank) for 2 hours. The sections were then washed three times for 10 min with PBS, and stained with an anti-mouse antibody (1:500, A-11005, Thermo fisher) for 1 hour, followed by another washing step. Excess liquid was then carefully removed and the sections were mounted with Fluoromount-G containing DAPI (0100-20, SouthernBiotech).

\section{mCherry-NLS and dystrophin/PCM1}

Following removal from $-80{ }^{\circ} \mathrm{C}$, sections from mCherry-NLS transfected muscle were allowed to equilibrate for $30 \mathrm{~min}$ at room temperature followed by $5 \mathrm{~min}$ fixation with freshly made $4 \%$ Formaldehyde (Thermo Fisher Scientific) in PBS pH 7.4. Immediately after fixation the slides were briefly rinsed with PBS pH 7.4 and washed three times 5 min PBS $\mathrm{pH}$ 7.4. The sections were then stained with an antibody against either dystrophin (1:200, ab15277, Abcam) or PCM1 (1:1000, HPA023370, Sigma) in a buffer containing $2 \%$ BSA in

This article is protected by copyright. All rights reserved. 
PBS pH 7.4, for 1 hour, followed by a 3 x 10 min washing step with PBS. The slides were stained with a secondary antibody (1:500, ab150077, Abcam) followed by a 3 x $10 \mathrm{~min}$ washing step before being mounted and processed as above (Figure 4).

PAX7

Satellite cells were labelled by fixing with $4 \%$ freshly made formaldehyde (Thermo Fisher Scientific) for $5 \mathrm{~min}$ followed by epitope retrieval using a sodium citrate/Tween buffer (10 $\mathrm{mM}, \mathrm{pH} 6.5,0.05 \%$ Tween 20 ) at $95{ }^{\circ} \mathrm{C}$ for $30 \mathrm{~min}$. Sections were stained overnight with antibody against PAX7 (Supernatant, Developmental Studies Hybridoma Bank) using a staining solution containing $50 \%$ supernatant diluted in PBS pH 7.4 supplemented with $2 \%$ BSA and $0.01 \%$ Triton X-100. Signal was obtained using the Tyramide Signal Enhancement (TSA) kit (Life Technologies) with a biotin-conjugated secondary antibody and streptavidinhorseradish peroxidase included within the kit. To visualize antibody-binding TSA-Alexa Fluor 594 was used. Sections were then counterstained with DAPI and PCM1 as described in previous sections (Figure 5).

\section{Microscopy and image analysis}

All images were visualized using a 40x or 60x PlanApo objective (NA 1.3 and 1.35, respectively) on a FluoView FV 1000 confocal microscope (Olympus) using the FV1000 software (version 1.7a). For analysis through the different focal planes, images were acquired in a stepwise manner through the z-plane with a step size of $1.0 \mu \mathrm{m}$ and a resolution of $800 \times 800$ pixels and laser scanning speed of 2,0 $\mu \mathrm{s} /$ pixel. For functional overload studies, images were captured with a resolution of 1024x1024 pixels and laser scanning speed of 2,0 $\mu$ s/pixel. 8-11 images were acquired from each muscle, and 8-22 fibres were analysed from each image. All fibres with a complete dystrophin ring inside the image were included, excluding fibres with central nuclei or inconsistent dystrophin ring due to focal plane differences. The total number of myonuclei in each of the included fibres was counted, including only myonuclei that were both PCM1 and DAPI positive, irrespective of the DAPIstaining appearing to be inside or outside the dystrophin ring. Cross-sectional area was measured on all included fibres. For comparing myonuclei identification with and without PCM1, nuclei in single focal planes was counted using the DAPI and dystrophin channel, using the mass centre inside the dystrophin inclusion criteria. The same images were also counted using PCM1 and the dystrophin channel. Images of PAX7 stained sections and

This article is protected by copyright. All rights reserved. 
transduced skeletal muscles were obtained using an Olympus BX-50WI compound microscope with a 40x (NA 0.3) water immersion objective. Images were acquired with an Andor iXion+ camera, controlled by Andor SOLIS software. Image processing was performed using ImageJ (NIH, USA), and image analysis was performed using Adobe Photoshop CS6 extended (Adobe Systems, USA). Analysis and 3D visualization was performed in Imaris 7.1 (Bitplane, USA)

\section{Western blotting}

For protein extraction, muscles were excised and snap-frozen in liquid nitrogen. The frozen tissue was crushed with a mortar cooled on dry ice, and resuspended in protein loading dye for the whole tissue analysis or PBS-based lysis buffer [PBS, pH 7.4, 1\% Triton X-100 (Sigma), 0.1\% Tween 20 (Sigma) with protease inhibitors (Complete Ultra EDTA-free tablets; Roche). The lysate was sonicated for 5 minutes in 30 seconds on and 30 seconds off cycles using a Bioruptor NextGen (Diagenode) at $5{ }^{\circ} \mathrm{C}$. For samples lysed in the PBS based buffer, the lysate was centrifuged for 5 minutes at $13.000 \mathrm{G}$ at $5{ }^{\circ} \mathrm{C}$. The soluble and insoluble fraction respectively was resuspended to equal volumes before subjected to SDS-

PAGE and immunoblotting as described previously ${ }^{31}$ using anti-PCM1 at a concentration of $1: 250$.

\section{Statistical procedures}

For the overload-experiment ( $\mathrm{n}=8$ rats), comparison of overloaded and contralateral control leg was calculated by Student's two-tailed unpaired t-test for both myonuclear number and cross-sectional area (CSA) using Graphpad Prism (version 7.0c). Statistical significance was accepted when $\mathrm{P}<0.05$.

\section{Conflict of interest}

None

\section{Funding}

Research council of Norway grant 240374

This article is protected by copyright. All rights reserved. 


\section{Acknowledgement}

We are grateful to Dr. Kristoffer Toldnes Cumming, Norwegian School of Sport Sciences, Oslo, Norway for providing the human cryosections for this study, and Kenth-Arne Hansson, Department of Biosciences, University of Oslo for technical assistance and feedback on our microscopical analysis.

\section{References}

1. Mauro, A: Satellite cell of skeletal muscle fibers. The Journal of biophysical and biochemical cytology, 9: 493-495, 1961.

2. Schmalbruch, H, Hellhammer, U: The number of nuclei in adult rat muscles with special reference to satellite cells. Anat Rec, 189: 169-175, 1977.

3. Utvik, JK, Nja, A, Gundersen, K: DNA injection into single cells of intact mice. Hum Gene Ther, 10: 291-300, 1999.

4. Bruusgaard, JC, Liestol, K, Ekmark, M, Kollstad, K, Gundersen, K: Number and spatial distribution of nuclei in the muscle fibres of normal mice studied in vivo. J Physiol, 551: 467-478, 2003.

5. Bruusgaard, JC, Liestol, K, Gundersen, K: Distribution of myonuclei and microtubules in live muscle fibers of young, middle-aged, and old mice. J Appl Physiol, 100: 20242030, 2006.

6. Bruusgaard, JC, Gundersen, K: In vivo time-lapse microscopy reveals no loss of murine myonuclei during weeks of muscle atrophy. J Clin Invest, 118: 1450-1457, 2008.

7. Bruusgaard, JC, Johansen, IB, Egner, IM, Rana, ZA, Gundersen, K: Myonuclei acquired by overload exercise precede hypertrophy and are not lost on detraining. Proc Natl Acad Sci U S A, 107: 15111-15116, 2010.

8. Egner, IM, Bruusgaard, JC, Eftestol, E, Gundersen, K: A cellular memory mechanism aids overload hypertrophy in muscle long after an episodic exposure to anabolic steroids. $J$ Physiol, 591: 6221-6230, 2013.

9. Egner, IM, Bruusgaard, JC, Gundersen, K: Satellite cell depletion prevents fiber hypertrophy in skeletal muscle. Development, 143: 2898-2906, 2016.

10. Gundersen, K, Bruusgaard, JC: Nuclear domains during muscle atrophy: nuclei lost or paradigm lost? J Physiol, 586: 2675-2681, 2008.

11. Bruusgaard, JC, Egner, IM, Larsen, TK, Dupre-Aucouturier, S, Desplanches, D, Gundersen, K: No change in myonuclear number during muscle unloading and reloading. J Appl Physiol, 113: 290-296, 2012.

12. Gundersen, K: Muscle memory and a new cellular model for muscle atrophy and hypertrophy. J Exp Biol, In press, 2016.

13. Gundersen, K: Muscle memory and a new cellular model for muscle atrophy and hypertrophy. J Exp Biol, 219: 235-242, 2016.

This article is protected by copyright. All rights reserved. 
14. Egner, IM, Bruusgaard, JC, Gundersen, K: An apparent lack of effect of satellite cell depletion on hypertrophy could be due to methodological limitations. Response to 'Methodological issues limit interpretation of negative effects of satellite cell depletion on adult muscle hypertrophy'. Development, 144: 1365-1367, 2017.

15. Goh, Q, Millay, DP: Requirement of myomaker-mediated stem cell fusion for skeletal muscle hypertrophy. eLife, 6, 2017.

16. Siu, PM, Pistilli, EE, Butler, DC, Alway, SE: Aging influences cellular and molecular responses of apoptosis to skeletal muscle unloading. Am J Physiol Cell Physiol, 288: C338-349, 2005.

17. Leeuwenburgh, C, Gurley, CM, Strotman, BA, Dupont-Versteegden, EE: Age-related differences in apoptosis with disuse atrophy in soleus muscle. Am J Physiol Regul Integr Comp Physiol, 288: R1288-1296, 2005.

18. Dupont-Versteegden, EE, Strotman, BA, Gurley, CM, Gaddy, D, Knox, M, Fluckey, JD, Peterson, CA: Nuclear translocation of EndoG at the initiation of disuse muscle atrophy and apoptosis is specific to myonuclei. Am J Physiol Regul Integr Comp Physiol, 291: R1730-1740, 2006.

19. Dammermann, A, Merdes, A: Assembly of centrosomal proteins and microtubule organization depends on PCM-1. The Journal of cell biology, 159: 255-266, 2002.

20. Hori, A, Toda, T: Regulation of centriolar satellite integrity and its physiology. Cell Mol Life Sci, 74: 213-229, 2017.

21. Villumsen, BH, Danielsen, JR, Povlsen, L, Sylvestersen, KB, Merdes, A, Beli, P, Yang, Y-G, Choudhary, C, Nielsen, ML, Mailand, N, Bekker-Jensen, S: A new cellular stress response that triggers centriolar satellite reorganization and ciliogenesis. The EMBO Journal, 32: 3029-3040, 2013.

22. Kubo, A, Tsukita, S: Non-membranous granular organelle consisting of PCM-1: subcellular distribution and cell-cycle-dependent assembly/disassembly. Journal of Cell Science, 116: 919-928, 2003.

23. Srsen, V, Fant, X, Heald, R, Rabouille, C, Merdes, A: Centrosome proteins form an insoluble perinuclear matrix during muscle cell differentiation. BMC Cell Biol, 10: $28,2009$.

24. Espigat-Georger, A, Dyachuk, V, Chemin, C, Emorine, L, Merdes, A: Nuclear alignment in myotubes requires centrosome proteins recruited by nesprin-1. Journal of Cell Science, 129: 4227-4237, 2016.

25. Bergmann, O, Zdunek, S, Alkass, K, Druid, H, Bernard, S, Frisen, J: Identification of cardiomyocyte nuclei and assessment of ploidy for the analysis of cell turnover. Exp Cell Res, 317: 188-194, 2011.

26. Hori, A, Barnouin, K, Snijders, AP, Toda, T: A non-canonical function of Plk4 in centriolar satellite integrity and ciliogenesis through PCM1 phosphorylation. EMBO Rep, 17: 326-337, 2016.

27. Hikida, RS, Van Nostran, S, Murray, JD, Staron, RS, Gordon, SE, Kraemer, WJ: Myonuclear loss in atrophied soleus muscle fibers. Anat Rec 247: 350-354, 1997.

28. Lee, SJ, Huynh, TV, Lee, YS, Sebald, SM, Wilcox-Adelman, SA, Iwamori, N, Lepper, C, Matzuk, MM, Fan, CM: Role of satellite cells versus myofibers in muscle hypertrophy induced by inhibition of the myostatin/activin signaling pathway.

This article is protected by copyright. All rights reserved. 
Proceedings of the National Academy of Sciences, 109: E2353-E2360, 2012.

29. Rosser, BW, Dean, MS, Bandman, E: Myonuclear domain size varies along the lengths of maturing skeletal muscle fibers. Int J Dev Biol, 46: 747-754, 2002.

30. Porter, GA: Dystrophin colocalizes with beta-spectrin in distinct subsarcolemmal domains in mammalian skeletal muscle. The Journal of cell biology, 117: 997-1005, 1992.

31. Eftestol, E, Egner, IM, Lunde, IG, Ellefsen, S, Andersen, T, Sjaland, C, Gundersen, K, Bruusgaard, JC: Increased hypertrophic response with increased mechanical load in skeletal muscles receiving identical activity patterns. Am J Physiol Cell Physiol, 311: C616-c629, 2016.

\section{Legends}

Figure 1: Identification of skeletal muscle myonuclei using PCM1 in mice

(a) Overview of a cross section from EDL muscle (single z-plane), stained with antibodies against dystrophin (red) to label individual fibre boundaries and PCM1 (green). Sections were counterstained with DAPI to label DNA (blue). (b) Arrow indicate two nuclei localized adjacent to each other, one being localized within the dystrophin ring and positively stained for PCM1. (c) 3D rendering showing the indicated nuclei being inside the dystrophin ring, co-localizing with PCM1 signal. Scale bar: $20 \mu \mathrm{m}$ for (a) and (b), $10 \mu \mathrm{m}$ for (c).

\section{Figure 2: Identification of skeletal muscle nuclei using PCM1 in adult humans and rat.}

(a) Cross section of human skeletal muscle, stained with antibodies against dystrophin (red) to label individual fibre boundaries, and PCM1 (green). Sections were counterstained with DAPI to label DNA (blue). (b) Single z-plane of a cross section of human skeletal muscle from confocal stacks split into its individual channels. Arrow indicates nuclei that appear to have the geometric centre outside the ring. (c) 3D rendering of the same area shown in (b), displaying the indicated nuclei (arrow) being inside the dystrophin ring. (d) 3D rendering of a confocal stack from rat EDL. Scale bar: (a) $40 \mu \mathrm{m}$ and (b, c, d) $20 \mu \mathrm{m}$.

\section{Figure 3: Quantification of myonuclear number using different methods}

(a) Number of nuclei assessed as myonuclei using either DAPI-positive with its mass centre inside the dystrophin ring or being PCM1/DAPI positive. Images (two per muscle) were assessed with (PCM1) and without (DAPI) the PCM1-channel in mice soleus ( $\mathrm{n}=3$ animals, 287 nuclei) and EDL ( $\mathrm{n}=3$ animals, 348 nuclei) muscles. Bars showing mean with $95 \% \mathrm{CI}$

(b) Micrographs from different z-planes illustrating how the same nucleus can appear both inside $(+4 \mu \mathrm{m})$ and outside $(0 \mu \mathrm{m}$ and $-1 \mu \mathrm{m})$ the dystrophin ring. (c) Longitudinal section

This article is protected by copyright. All rights reserved. 
showing local bulging of the dystrophin around the myonucleus. Scale bar: $20 \mu \mathrm{m}$ for (b) and $10 \mu \mathrm{m}$ for (c).

\section{Figure 4: Identification of skeletal muscle nuclei by in vivo transduction of ACTA1- mCherry-NLS in mice.}

(a) Plasmid chart showing the expression cassette used for generating the adeno-associated viral serotype 6 vectors for in vivo transduction of mice skeletal muscle. (b) Image of a single z-plane showing transduced skeletal muscle nuclei (red) expressing mCherry-NLS (arrows) within the dystrophin boundary (green). Cross sections were counterstained with DAPI to label DNA (blue). (c) Image of a single z-plane from a muscle cross section expressing mCherry-NLS counterstained with DAPI and antibodies against PCM1 to show colocalization of mCherry-NLS and PCM1 signal. (d) Immunoblot of PCM1(arrow) in whole tissue lysate (left) and in soluble and insoluble fraction (right). Scale bar: $20 \mu \mathrm{m}$ for (b) and (c).

\section{Figure 5: PCM1 was not detected in satellite cells}

Image showing a single z-plane from a cross section of the plantaris muscle overloaded for two weeks stained with an antibody against PAX7 (red) to visualize satellite cells showing no co-localization with PCM1 signal (green). Sections were counterstained with DAPI to visualize DNA (blue). Scale bar is $40 \mu \mathrm{m}$.

\section{Figure 6: PCM1 stained newly inserted myonuclei after overload-induced hypertrophy}

(a) Image showing a single z-plane of cross sections from rat soleus muscle after 14 days of overload (bottom), with its respective contralateral control (top). Sections were stained with antibodies against dystrophin (red) to label individual fibre boundaries, DAPI (blue) to label DNA and PCM1 (green). Scale bar is $100 \mu \mathrm{m}$. (b) Number of myonuclei (top) and crosssectional area (CSA, bottom) per fibre cross section in control ( $\mathrm{n}=8$ rats, 115-173 fibres per rat) and overloaded soleus muscle ( $\mathrm{n}=8$ rats, 93-151 fibres). $*(\mathrm{p}<0.05), * *(\mathrm{p}<0.01)$

Significantly different from contralateral control. Bars showing mean with $95 \%$ CI, circles and corresponding lines showing muscle average values in contralateral and overloaded muscle within a single rat.

This article is protected by copyright. All rights reserved. 
(a)

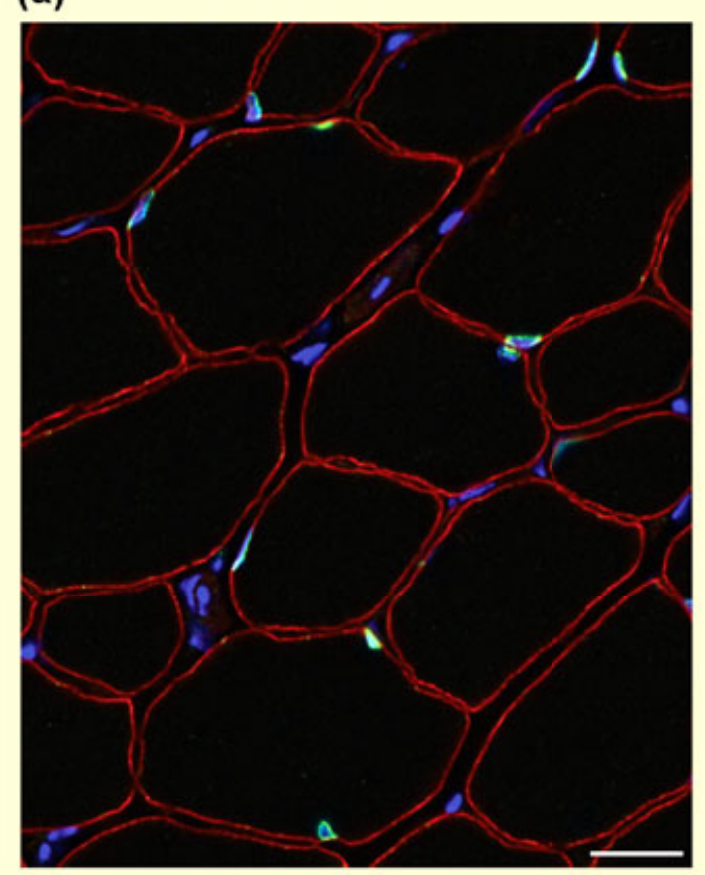

(b)

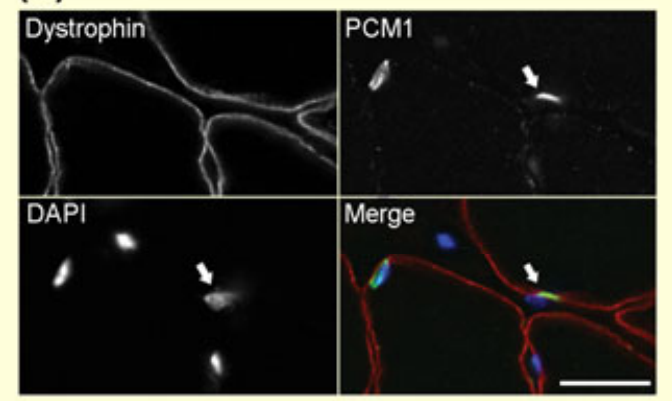

(c)

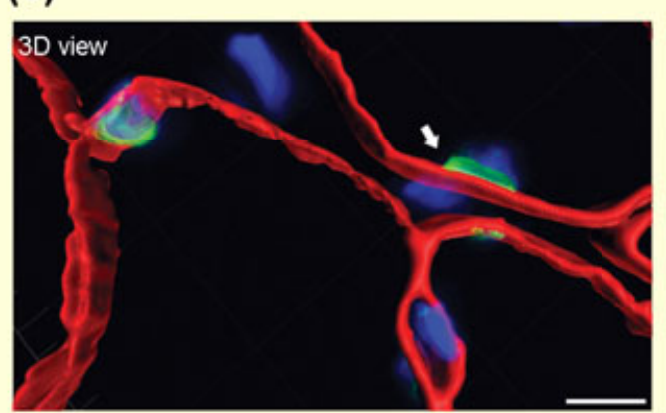


(a)

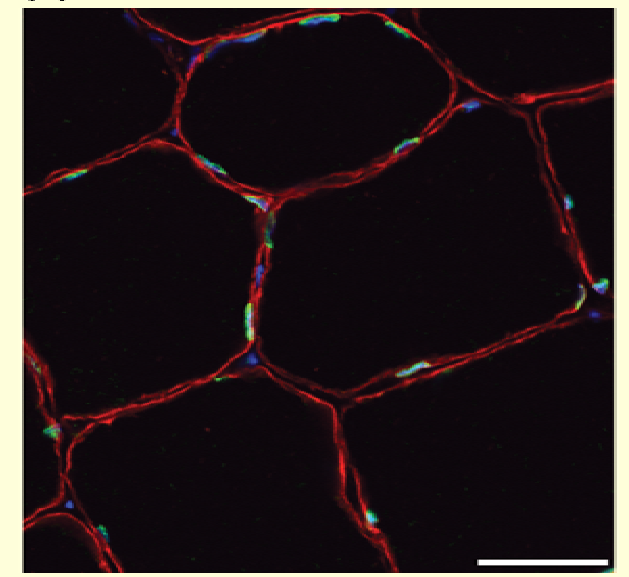

(c)

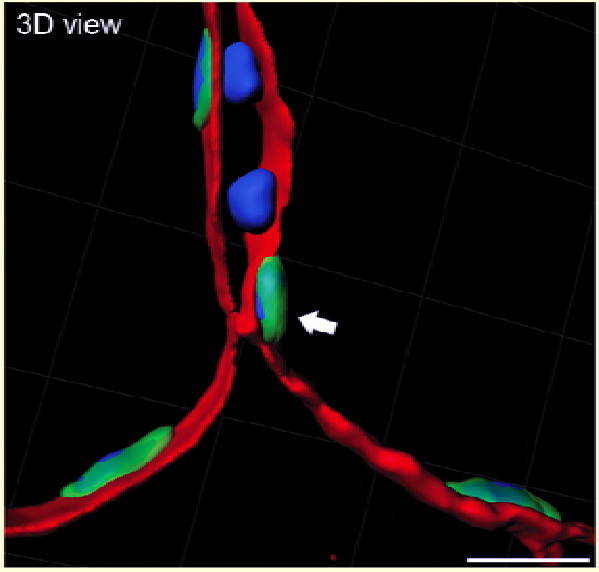

(b)

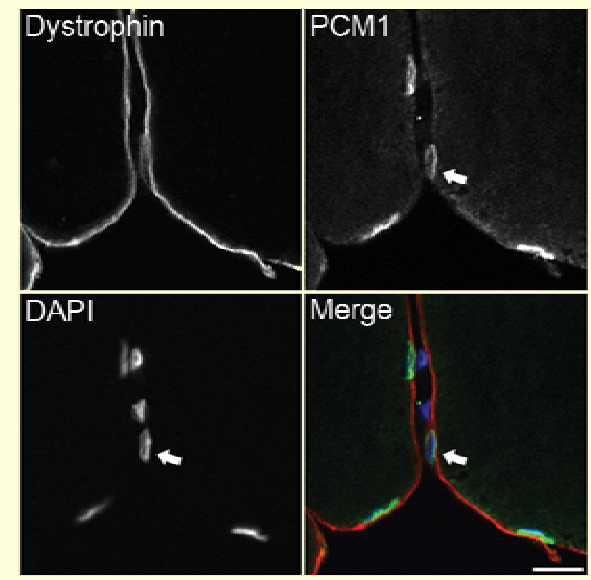

(d)

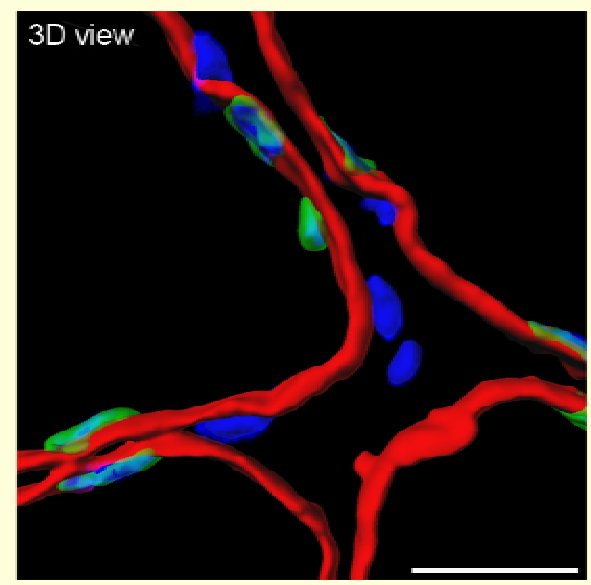


(a)

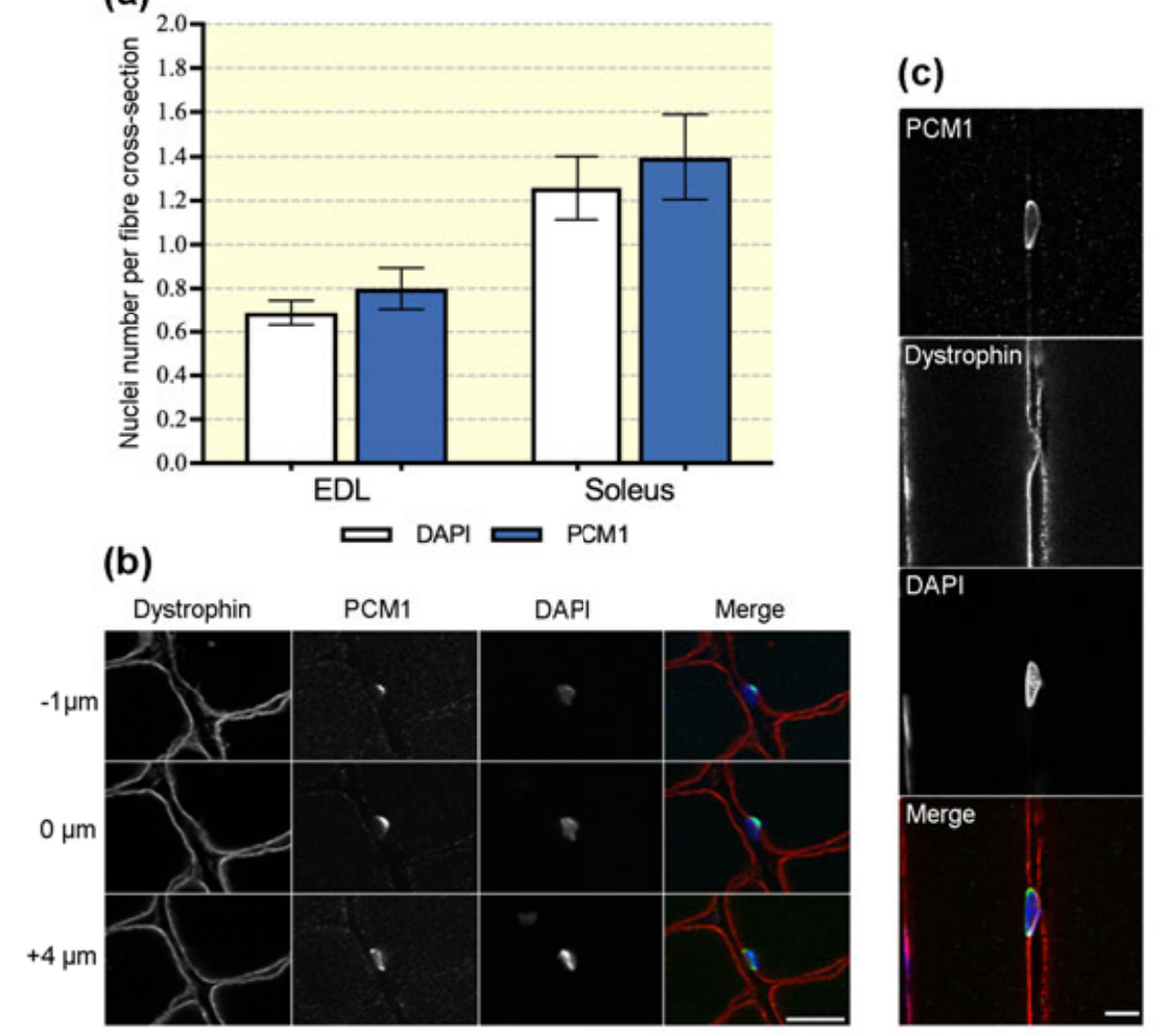


(a)

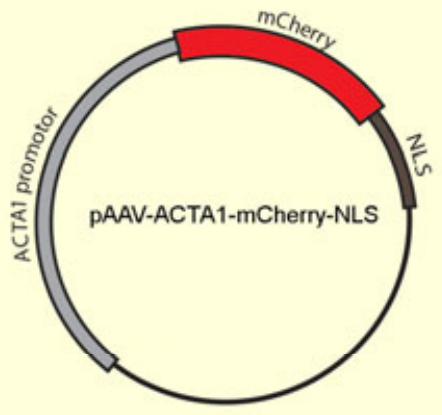

(c)

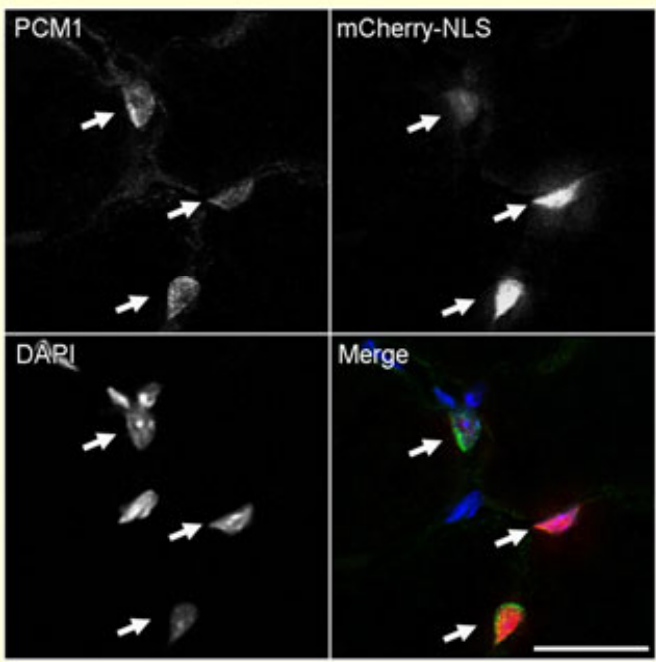

(b)

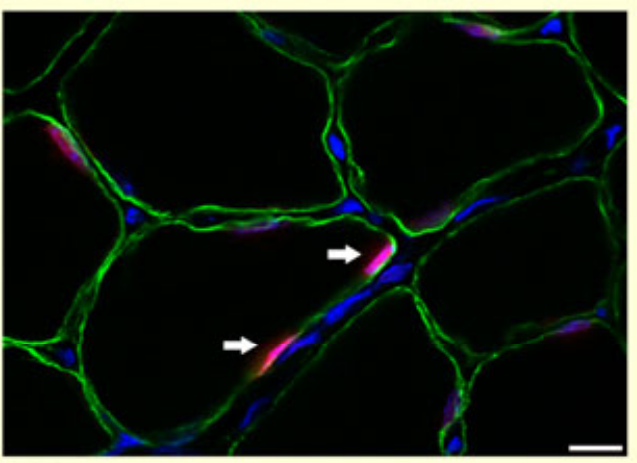

(d)

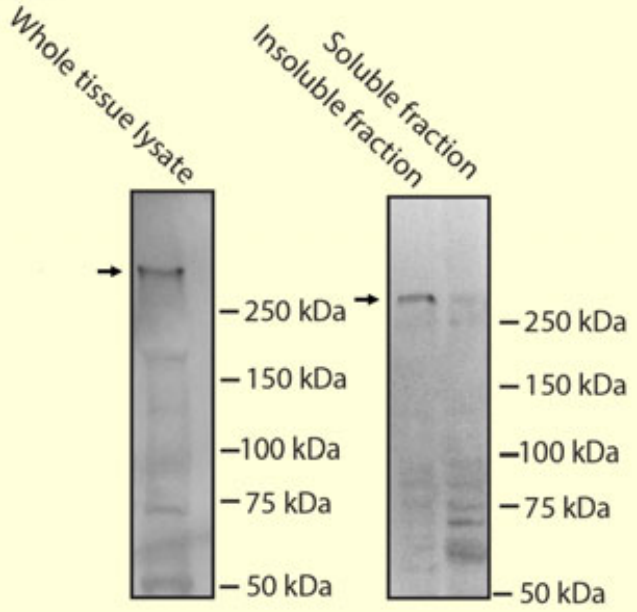

This article is protected by copyright. All rights reserved. 

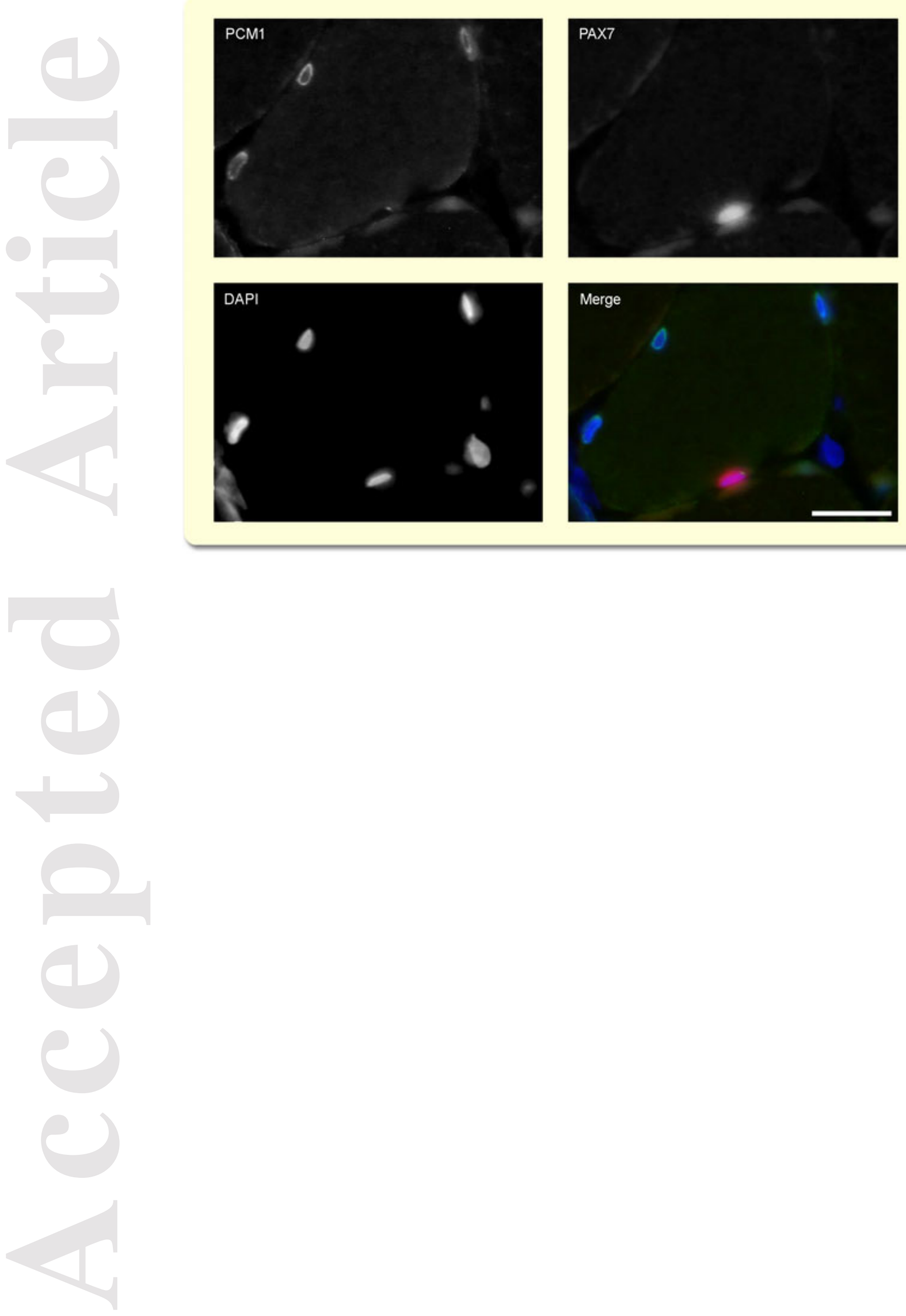
(a)
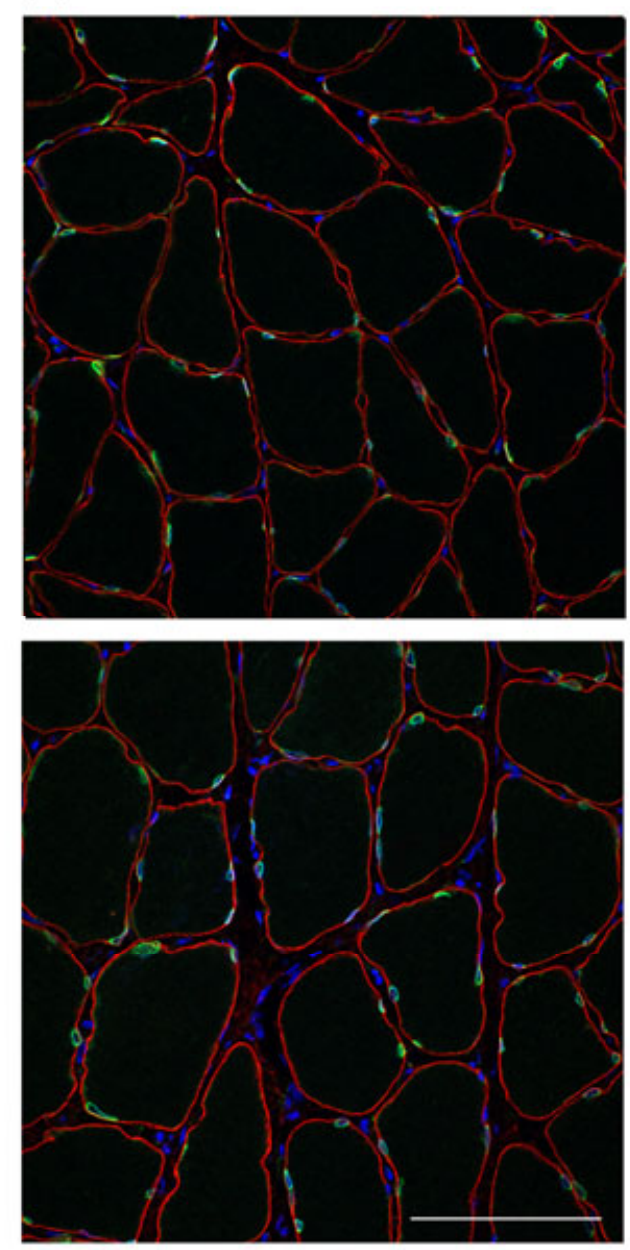

(b)
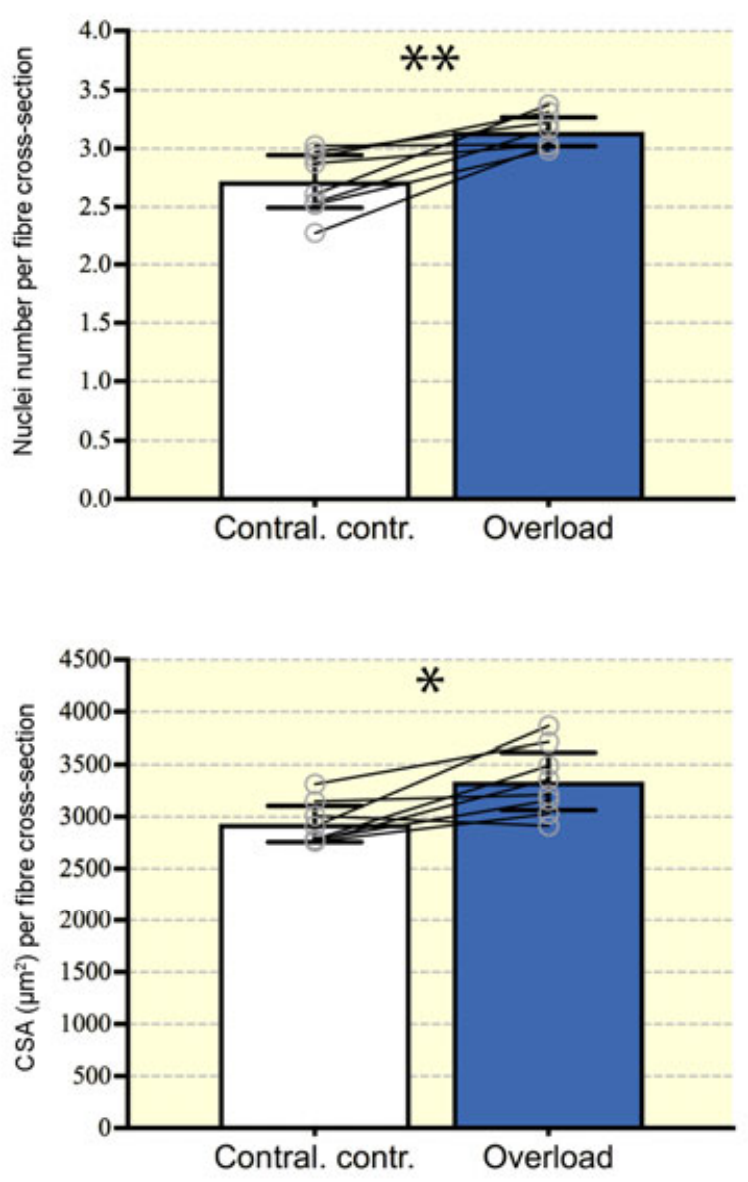

This article is protected by copyright. All rights reserved. 